\title{
A Note on Dynamic Data Driven Wildfire Modeling
}

\author{
J. Mandel ${ }^{1}$, M. Chen ${ }^{1}$, L.P. Franca ${ }^{1}$, C. Johns ${ }^{1}$, A. Puhalskii ${ }^{1}$, J.L. Coen ${ }^{2}$, \\ C.C. Douglas ${ }^{3}$, R. Kremens ${ }^{4}$, A. Vodacek ${ }^{4}$, and W. Zhao ${ }^{5}$ \\ ${ }^{1}$ University of Colorado Denver, Denver, CO 80217-3364, USA \\ ${ }^{2}$ National Center for Atmospheric Research, Boulder, CO 80307-3000, USA \\ ${ }^{3}$ University of Kentucky, Lexington, KY 40506-0045, USA \\ ${ }^{4}$ Rochester Institute of Technology, Rochester, NY 14623-5603, USA \\ ${ }^{5}$ Texas A\&M University, College Station, TX 77843-1112, USA
}

\begin{abstract}
A proposed system for real-time modeling of wildfires is described. The system involves numerical weather and fire prediction, automated data acquisition from Internet sources, and input from aerial photographs and sensors. The system will be controlled by a non-Gaussian ensemble filter capable of assimilating out-of-order data. The computational model will run on remote supercomputers, with visualization on PDAs in the field connected to the Internet via a satellite.
\end{abstract}

\section{Introduction}

Today, there exists virtually no capability to predict the spread of wildfires. In 2000 alone (the worst fire season in over 50 years), over 90,000 wildfires cost an estimated $\$ 10$ billion in suppression and lost resources. Current field tools for diagnosing expected fire behavior are simple algorithms that can be run on simple pocket calculators. Researchers and fire managers alike envision a future when they can rely on complex simulations of the interactions of fire, weather, and fuel, driven by remote sensing data of fire location and land surface properties. And get the results as easy to understand animations on a small laptop or PDA, have the computer incorporate all information as soon as it becomes available, and assess the likelihood of possible scenarios. This is how computers work in the imagination of sci-fi movie directors. This project is to build a prototype as the first step to make it a reality.

This note is based on [24], where further details can be found.

\section{Overview of the Proposed System}

The objective of this project is to develop a prototype hardware and software system to integrate available information related to a wildfire in progress, and to provide a numerical prediction of the wildfire in a visual form, including tools to predict the outcome of firefighting strategies. The proposed system will have the following main components: 
- Numerical coupled atmosphere/fire model

- Data acquisition (measurements)

○ From Internet: maps (GIS), aggregated fire information, weather

○ Field information: aerial photos, sensors

- Visualization and user interface

- Dynamic Data Assimilation control module

- Guaranteed secure communication infrastructure

The numerical model accepts data in a mesh format. The Dynamic Data Assimilation control module will call the numerical model to execute multiple simulations, extract data from the state of the numerical model to be compared with the measurements and modify the state of the numerical model to match the measurements. The visualization and user interface module will display the results of the simulation and support user input to control alternative firefighting scenarios.

The numerical model will run on one or more remote supercomputers, while the visualization and user interface will run on PDAs in the field. Software agents and search engines will provide internet data, while networked sensors and cameras in airplanes will provide the field data. The field internet connection will be implemented using a local wireless network, bridged to the internet by a consumergrade cheap broadband satellite connection and satellite data phones.

\section{Weather and Fire Model}

NCAR's coupled atmosphere-fire model is described in detail in [9, 12, 13, 14]. A 3D atmospheric prediction model $[7,8,10,11]$ has been coupled with an empirical fire spread model $[1,2,29,30]$ such that the heat fluxes from the fire feed back to the atmosphere to produce fire winds, while the atmospheric winds drive the fire propagation. This wildfire simulation model can thus represent the complex interactions between a fire and local weather.

The existing numerical model is a legacy FORTRAN code that took a quarter of century and substantial scientific expertise to develop. We are proceeding in these steps: encapsulate execution of one time step as a subroutine; define and enforce software interfaces; upgrade the fire model from empirical to differential equations based; and speed up the model using techniques such as OpenMP and multigrid.

The overall system will run many instances of the model simultaneously; we expect that each instance of the model will run on a single CPU or an SMP node.

Our next step up from an empirical model of fire propagation is a model based on a reaction-convection-diffusion equation describing simplified fire physics with additional stochastic terms, which also model spotting (secondary fires started by flying embers). For related models, see [4, 16, 22]. There are two coupled equation: one for the temperature, derived from the balance of heat fluxes, and one equation for the rate of consumption of the fuel. The temperature influences the burn rate, which in turn determines the term for the heat flux generated by the burning in the equation for the temperature. Added stochastic terms are a noise expressing the uncertainties of the model, and flight of burning embers, modeled by replacing (with a certain probability, dependent on the temperature and the wind) the temperature a point by the temperature at another randomly selected point. 
Anticipated developments of the fire model include several species of fuel to model different types of fire (grass, brush, crown fire), spotting terms with delay to account for the time it takes for the fire started by the flying embers to become visible on the computational grid scale, and of course coupling with the NCAR atmosphere model.

\section{Data Acquisition}

Maps, sometimes including fuel information, are available from GIS files maintained by public agencies as well as private companies. We are working on translating the GIS format files into meshed data suitable for input into the model. Raw as well as assimilated weather data are readily available from numerous sources on the Internet, including NOAAPORT broadcast, MesoWest weather stations, and the Rapid Update Cycle (RUC) weather system by NOAA. Aggregated fire information is available from the GeoMAC project at USGS. The challenge here is to develop intelligent converters of the information, which can deal with the constantly changing nature of Internet sites.

Thermal and near infrared images obtained from a manned or unmanned airborne platform is perhaps one of the best means of tracking the advance of wildland fires [28, 32]. Infrared radiation will pass through smoke relatively unattenuated, thus providing a signal of the exact fire location.

To obtain the geographic coordinates for the ground locations of individual pixels in an image that may correspond to the fire front or hotspot, the location and the direction that the camera was pointing at the time of image capture can be obtained by a combination of GPS readings for aircraft position and 3-axis inertial measurement unit data to determine the pointing direction of the camera. Terrain induced distortion can be corrected by parallax measurements on overlapping stereo images, or from given knowledge of the terrain and the camera pointing direction and altitude [25]. While topographic maps exist for much of the U.S., one very promising data set for the U.S. and much of the rest of the world is from the Shuttle Radar Topography Mission [27].

Networked, autonomous environmental detectors may be placed in the vicinity of a fire for point measurements of fire and weather information [21]. For geolocation, the units can either be built with a GPS capability or a less expensive approach is to plan for a GPS position to be taken at the exact location of the deployment using an external GPS unit and uploading the position data to the detector memory.

\section{Dynamic Data Assimilation}

An important feature of DDDAS is that the model is running all the time and it incorporates new data as soon as it arrives. Also, in this application, uncertainty is dominant because important processes are not modeled, there are measurement and other errors in the data, the system is heavily nonlinear and ill-posed, and there are multiple possible outcomes. This type of problem is a natural candidate for sequential Bayesian filtering (sequential because the data is incorporated sequentially as it 
arrives rather than all at once). The state of the system at any time period is represented by a collection of physical variables and parameters of interest, usually at mesh points. To be able to inject data arriving out of sequence, we will work with the time-state vector $x$, which will contain snapshots of system states at different points in time. The knowledge of the time-state of the system is represented in the model as a probability density function $p(x)$. The model will represent the probability distribution using an ensemble of time-state vectors $x_{1}, \ldots, x_{n}$. Thus, the number of the system states maintained by the model will be equal to the number of time snapshots saved for updating out of order data multiplied by the ensemble size, a product that will easily reach the thousands. Each of these system states will be advanced in time via separate simulations and substantial parallel supercomputing power will be required.

Sequential filtering consists of successive updating of the model state using data via Bayes theorem. The current state of the model is called the prior or the forecast probability density $p^{f}(x)$. The arriving data consists of measurements $y$ along with an information how the measured quantities are derived from the system state $x$, and information about the distribution of measurement errors. That is, the additional information provided by the data to the model is represented by the vector $y$ and a conditional probability density function $p(y \mid x)$. The update is incorporated into the model using the Bayes theorem, resulting in the posterior or analysis probability density

$$
p^{a}(x)=\frac{p(y \mid x) p^{f}(x)}{\int p(y \mid \xi) p^{f}(\xi) d \xi},
$$

which then becomes the new state of the model. The model states continue advancing in time, with a corresponding advancement of the probability density, until new data arrives. The system states for times within the time stamps of the data are system estimation. The system states beyond the time stamp of the data constitute a prediction. Note that advancing the model in time and injecting the data into the model is decoupled.

Clearly, the crucial question is how the probability distributions are represented in the model. The simplest case is to assume normal distributions for the states $x$ and the conditional distributions $p(y \mid x)$ and that the observations $y$ depend linearly upon the states. These assumptions give the well-known Kalman filter formulas [20]. In particular, the posterior is also normal with the mean equal to the solution $x^{a}$ of the least squares problem

$$
\min _{x^{a}}\left\{\left(x^{a}-x^{f}\right)^{T} \Sigma^{-1}\left(x^{a}-x^{f}\right)+\left(y-H x^{a}\right)^{T} R^{-1}\left(y-H x^{a}\right)\right\} .
$$

Ensemble Kalman filters [17, 18, 19, 31] represent a normal distribution by a sample, which avoids manipulation of covariance matrix $\Sigma$, replacing it with sample covariance estimate. Thus, the ensemble approach is particularly useful in problems where the state vector $x$ or the observation vector $y$ are large. However, existing methods do not support out of order data, because they operate on only one time snapshot, and extensions of ensemble filters to the time-state model described here have to be worked out. Further, if the time evolution function $f$ is nonlinear or the observation matrix $H$ in (2) is replaced by a nonlinear function, the probability 
distribution $p(x)$ is not expected to be normal. Particle filters [3, 15] can approximate any probability distribution, not only normal, by a sample of vectors $x_{1}, \ldots, x_{n}$, with corresponding weights $w_{1}, \ldots, w_{n}$; then approximately $x \sim D$, where the probability of any event $\omega$ is calculated by

$$
D(\omega)=\sum_{x_{i} \in \omega} w_{i} .
$$

Time-state models have been used in processing out-of-order data by particle filters [23]. But the approximation (3) is rather crude and, consequently, particle filters generally require large samples, which is not practical in our application. For this reason, we are investigating data assimilation by ensemble filters based on Gaussian mixtures $[5,6]$, where the probability distributions are approximated by a weighted mixture of normal distributions, such as

$$
x \sim N\left(\mu_{i}, \Sigma_{i}\right) \text { with probability } p_{i} .
$$

To assimilate data that depends on system state in a highly nonlinear way, such as fire perimeters from aerial photographs, one of the options we are investigating is replacing the least squares problem (2) by the nonlinear optimization problem

$$
\min _{x^{a}}\left\{\left(x^{a}-x^{f}\right)^{T} \Sigma^{-1}\left(x^{a}-x^{f}\right)+\left(y-h\left(x^{a}\right)\right)^{T} R^{-1}\left(y-h\left(x^{a}\right)\right)\right\},
$$

which may have multiple local minima. Alternatively, we consider updating the model state by minimizing some probabilities that the solution is off by more than a given amount, e.g.,

$$
\min _{x^{a}}\left\{P\left(\neg\left|x^{a}-x^{f}\right|<\varepsilon_{x} \vee \neg\left|y-h\left(x^{a}\right)\right|<\varepsilon_{y}\right)\right\},
$$

where the inequalities are understood term by term, and $\varepsilon_{x}, \varepsilon_{y}$ are vectors of tolerances. This type of threshold estimates, related to Bahadur efficiency [26], could be quite important in fire problems, because of the nature of fires. Unlike in the case when the distribution of $x$ is normal, here a least squares estimate may say little about whether or not the ignition temperature in a given region has been reached.

The Dynamic Data Assimilation module needs also to steer the data acquisition. In terms of the Bayesian update, one method of steering would be to select the measurements to minimize the variance of the posterior distribution. In the linear Gaussian case, this becomes an optimization problem for the observation matrix $H$ under the constraints of what measurements can be made.

Finally, guaranteed secure communication [33] delivers messages within a preset delay with a given probability close to one. This means that loss of data or loss of communication with ensemble members could be, in principle, handled as a part of the stochastic framework, and the Dynamic Data Assimilation module should take advantage of that.

Acknowledgments. This research has been supported in part by the National Science Foundation under grants ACI-0325314, ACI-0324989, ACI-0324988, ACI-0324876, and ACI-0324910. 


\section{References}

1. Albini, F. A.: PROGRAM BURNUP: A simulation model of the burningof large woody natural fuels. Final Report on Research Grant INT-92754-GR by U.S.F.S. to Montana State Univ., Mechanical Engineering Dept. (1994)

2. Anderson, H.: Aids to determining fuel models for estimating fire behavior. USDA Forest Service, Intermountain Forest and Range Experiment Station, Report INT-122 (1982)

3. Arulampalam, M., Maskell, S., Gordon, N., Clapp, T.: A tutorial on particle filters for online nonlinear/non-Gaussian Bayesian tracking, IEEE Transactions on Signal Processing, 50 (2002) 174-188

4. Asensio, M. I., Ferragut, L.: On a wildland fire model with radiation, Int. J. Numer. Meth. Engrg, 54 (2002) 137-157

5. Bengtsson, T., Snyder, C., Nychka, D.: A nonlinear filter that extends to high dimensional systems. J. of Geophys. Res. - Atmosphere, in review (2003)

6. Chen , R., Liu, J. S.: Mixture Kalman filters, J. of the Royal Statistical Society: Series B, 62 (2000) 493-508

7. Clark, T. L.: A small-scale dynamic model using a terrain-following coordinate transformation, J. of Comp. Phys., 24 (1977) 186-215

8. Clark, T. L.: Numerical simulations with a three-dimensional cloud model: lateral boundary condition experiments and multi-cellular severe storm simulations, J. Atmos. Sci., 36 (1979) 2191-2215

9. Clark, T. L., Coen, J., Latham, D.: Description of a coupled atmosphere-fire model, Intl. J. Wildland Fire, 13, in print (2004)

10. Clark, T. L., Hall, W. D.: Multi-domain simulations of the time dependent Navier- Stokes equations: Benchmark error analysis of some nesting procedures, J. of Comp. Phys., 92 (1991) 456-481

11. Clark, T. L., Hall, W. D.: On the design of smooth, conservative vertical grids for interactive grid nesting with stretching, J. Appl. Meteor., 35 (1996) 1040-1046

12. Clark, T. L., Jenkins, M. A., Coen, J., Packham, D.: A coupled atmospheric-fire model: Convective feedback on fire line dynamics, J. Appl. Meteor, 35 (1996) 875-901

13. Clark, T. L., Jenkins, M. A., Coen, J., Packham, D.: A coupled atmospheric-fire model: Convective Froude number and dynamic fingering, Intl. J. of Wildland Fire, 6 (1996) 177190

14. Coen, J. L., Clark, T. L., Latham, D.: Coupled atmosphere-fire model simulations in various fuel types in complex terrain, in 4th. Symp. Fire and Forest Meteor. Amer. Meteor. Soc., Reno, Nov. 13-15 (2001) 39-42

15. Doucet, A., Freitas, N. de, Gordon, N. (ed.): Sequential Monte Carlo in Practice, Springer.

16. Dupuy, J. L. and Larini, M. (1999). Fire spread through a porous forest fuel bed: A radiative and convective model including fire-induced flow effects, Intl. J. of Wildland Fire, 9 (2001) 155-172.

17. Evensen, G.: Sequential data assimilation with nonlinear quasi-geostrophic model using Monte Carlo methods to forecast error statistics, J. Geophys. Res.,99 (C5) (1994) 143-162

18. Evensen, G.: The ensemble Kalman filter: Theoretical formulation and practical implementtation. http://www.nersc.no/geir (2003)

19. Houtekamer, P., Mitchell, H. L.: Data assimilation using an ensemble Kalman filter technique, Monthly Weather Review, 126 (1998) 796-811

20. Jazwinski, A. H.: Stochastic processes and filtering theory, Academic Press, New York (1970)

21. Kremens, R., Faulring, J., Gallagher, A.,Seema, A., Vodacek, A.: Autonomous fielddeployable wildland fire sensors, Intl. J. of Wildland Fire, 12 (2003) 237-244

22. Linn, R. Reisner, J.,Colman, J., Winterkamp, J.: Studying wildfire behavior using FIRETEC, Int. J. of Wildland Fire, 11 (2002) 233-246 
23. Mallick, M., Kirubarajan, T., Arulampalam, S.: Out-of-sequence measurement processing for tracking ground target using particle filters, in Aerospace Conference Proceedings, vol. 4, IEEE (2002) 4-1809-4-1818

24. Mandel, J., Chen, M., Franca, L.P., Johns, C., Puhalskii, A., Coen, J.L., Douglas, C.C., Kremens, R., Vodacek, A., Zhao, W.: Dynamic data driven wildfire modeling, F. Darema (ed.), Dynamic Data Driven Applications Systems, Klumer Academic Publishers (2004)

25. Mostafa, M., Hutton, J., Lithopoulos, E.: Covariance propagation in GPS/IMU - directly georeferenced frame imagery, in Proceedings of the Asian Conference on Remote Sensing 2000, Taipei, Center for Space; Remote Sensing Research, National Central University and Chinese Taipei Society of Photogrammetry and Remote Sensing (2000)

26. Puhalskii, A., Spokoiny, V.: On large-deviation efficiency in statistical inference, Bernoulli, 4 (1998) 203-272

27. Rabus, B. Eineder, M. Roth, A., Bamler, R.: The shuttle radar topography mission - a new class of digital elevation models acquired by spaceborne radar, Photogrammetric Engineering and Remote Sensing, 57 (2003) 241-262

28. Radke, L. R., Clark, T.L., Coen, J. L., Walther, C., Lockwood, R. N., Riggin, P., Brass, J., Higgans, R.: The wildfire experiment (WiFE): Observations with airborne remote sensors, Canadian J. Remote Sensing, 26 (2000) 406-417

29. Richards, G. D.: The properties of elliptical wildfire growth for time dependent fuel and meteorological conditions, Combust. Sci. and Tech., 95 (1994) 357-383

30. Rothermel, R. C.: A mathematical model for predicting fire spread in wildland fires. USDA Forest Service Research Paper INT-115 (1972)

31. Tippett, M. K., Anderson, J. L., Bishop, C. H., Hamill, T. M., Whitaker, J. S.: Ensemble square root filters, Monthly Weather Review, 131 (2003) 1485-1490

32. Vodacek, A., Kremens, R. L., Fordham, A. J., VanGorden, S. C., Luisi, D., Schott, J. R.: Remote optical detection of biomass burning using a potassium emission signature, Intl. J. of Remote Sensing, 13 (2002) 2721-2726

33. Zhao, W.: Challenges in design and implementation of middlewares for real-time systems, J. of Real-Time Systems, 20 (2001) 1-2 